\title{
Strangulated Transomental Hernia with Intestinal Necrosis: An Autopsy Case
}

Satoshi Furukawa*, Lisa Wingenfeld, Ikuo Sakaguchi, Tokiko Nakagawa, Akari Takaya, Satomu Morita, Shigeru Yamasaki and Katsuji Nishi Department of Legal Medicine, Shiga University of Medical Science, Otsu, Shiga, Japan

\begin{abstract}
Transomental openings are usually congenital. Transomental hernia is a rare cause of intestinal obstruction. A 36-year-old female was found to have a transomental hernia at autopsy. We report the case of the strangulated intestinal obstruction with no past history.
\end{abstract}

Keywords: Transomental hernia; Strangulated intestinal obstruction; Autopsy

\section{Introduction}

Internal hernias result from the protrusion of one or more abdominal viscera through an intra-peritoneal opening, with the herniated viscera remaining inside the abdominal cavity. Predisposing factors for transomental hernias include congenital anatomic defects of the liver, lesser sac, mesentery, as well as the presence of adhesions or increased intra-abdominal pressure [1,2]. Abnormal transomental openings are usually congenital, and rarely traumatic or iatrogenic [3]. Internal hernias are infrequent, accounting for 0.2 to 0.9 of the cases of intestinal obstruction, and lead to 0.5 to $4.1 \%$ of the cases of acute intestinal obstruction caused by hernias [4-6]. Transomental hernias through the greater or lesser omentum are even rarer, representing 1 to $4 \%$ of all internal hernias [3].

\section{Case Report}

A 36-year-old female who had previously been in good health, was admitted to the Emergency Department, complaining of having had diffuse abdominal pain and vomiting. At autopsy, she was found to have a transomental hernia. A loop of small bowel $120 \mathrm{~cm}$ in length had prolapsed in a posterior-anterior direction through a $2 \mathrm{~cm}$ hole in the greater omentum. The ileal segment was strangulated and upon widening the hernia orifice, the ischemic bowel was removed. (Figure $1,2)$

\section{Discussion}

Transomental hernia is a rare cause of intestinal obstruction and accounts for less than $1 \%$ of internal hernias [7]. The exact aetiology is unknown, although it has been proposed congenital in origin [8]. Transomental hernia has a defect which allows bowel to pass through the omentum. In the presence of necrosis, perforation or irreversible ischemia, intestinal resection is performed [6]. The extreme difficulty

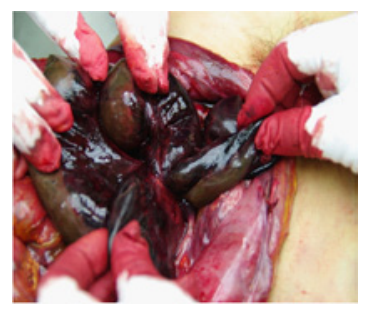

Figure 1: Widened Hernia Orifice.

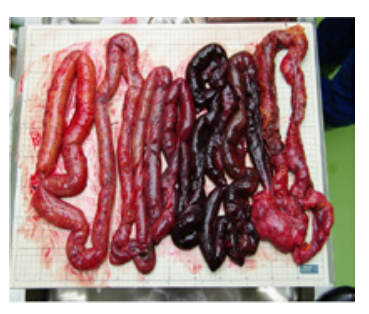

Figure 2: Removed Ischemic Bowel.

in making diagnoses of specific signs and symptoms is emphasized [9] On the other hand, internal hernias are invariably manifested as acute intestinal obstruction that requires early diagnosis and immediate surgery. Unfortunately, there were no diagnostic, clinical or radiological findings in this case. We reported the case of the strangulated intestinal obstruction with no past history.

\section{References}

1. Yasuda S, Inatsugi N, Sakurai T, Nakamura H, Hashimoto T, et al. (1989) A case of intestinal obstruction due to a hernia traversing the lesser sac. Jpn J Surg 19: 70-73.

2. Pessaux P, Tuech JJ, Derouet N, Du Plessis R, Ronceray J, et al. (1999) Internal hernia: a rare cause of intestinal obstruction. Apropos of 14 cases. Ann Chir 53: 870-873.

3. Gulino D, Giordano O, Gulino E (1993) Les hernias internes de l'abdomen. A propos de 14 cas. J Chir 130: 179-195.

4. Ghahremani GG (1984) Internal abdominal hernias. Surg Clin North Am 64: 393-406.

5. Sufian S, Matsumoto T (1975) Intestinal obstruction. Am J Surg 130: 9-14

6. Ozenc A, Ozdemir A, Coskun T (1998) . . Int Surg 83: 167-170.

7. Stewart JOR (1962) Transepiploic hernia. British Journal of Surgery 49: 649 652 .

*Corresponding author: Satoshi Furukawa, Department of Legal Medicine, Shiga University of Medical Science, Setatsukinowa, Otsu City, Shiga 520-2192, Japan, Tel: +81-77-548-2200; E-mail: 31041220@belle.shiga-med.ac.jp

Received June 19, 2012; Accepted August 22, 2012; Published August 30, 2012

Citation: Furukawa S, Wingenfeld L, Sakaguchi I, Nakagawa T, Takaya A, et al (2012) Strangulated Transomental Hernia with Intestinal Necrosis: An Autopsy Case. J Forensic Res 3:163. doi:10.4172/2157-7145.1000163

Copyright: (c) 2012 Furukawa S, et al. This is an open-access article distributed under the terms of the Creative Commons Attribution License, which permits unrestricted use, distribution, and reproduction in any medium, provided the original author and source are credited. 
Citation: Furukawa S, Wingenfeld L, Sakaguchi I, Nakagawa T, Takaya A, et al. (2012) Strangulated Transomental Hernia with Intestinal Necrosis: An Autopsy Case. J Forensic Res 3:163. doi:10.4172/2157-7145.1000163

Page 2 of 2

8. Treves F (1884) Intestinal Obstructions: its varieties with their pathology, diagnoses and treatment. Cassel London 28-31.
9. Genovese AM, Taranto F, Fiore D, Segreto M, Giardinelli A, et al. (2000) Internal abdominal hernia. Unusual cause of intestinal occlusion. Minerva Chir 55: $177-180$ 